Journal of Animal and Veterinary Advances 11 (10): 1538-1545, 2012

ISSN: $1680-5593$

(C) Medwell Journals, 2012

\title{
Different Duration of Cold Stress Enhances Pro-Inflammatory Cytokines Profile and Alterations of Th1 and Th2 Type Cytokines Secretion in Serum of Wistar Rats
}

\author{
${ }^{1}$ Jing Ru Guo, ${ }^{2} \mathrm{Shi} \mathrm{Ze} \mathrm{Li},{ }^{3} \mathrm{Hong}$ Gang Fang, ${ }^{2} \mathrm{Xu}$ Zhang, ${ }^{1} \mathrm{Jian}$ Fa Wang, \\ ${ }^{2}$ Shuang Guo, ${ }^{1,}{ }^{2}$ Hong JI, ${ }^{2}$ Lin Zang, ${ }^{2} \mathrm{Li}$ Guo, ${ }^{2} \mathrm{Li}$ Zhen and ${ }^{2}$ Huan Min Yang \\ ${ }^{1}$ College of Animal Science and Veterinary Medicine, Jilin University, \\ 130062 Changchun, Jilin Province, P.R. China \\ ${ }^{2}$ College of Animal Science and Veterinary Medicine, \\ Heilongjiang Bayi Agricultural University, \\ ${ }^{3}$ Test Analysis Laboratory, Daqing Oilfield General Hospital, \\ 163319 Daqing, Heilongjiang Province, P.R. China
}

\begin{abstract}
Cold stress is generally considered to suppress the immune system and may lead to an increase in the occurrence of disease in the presence of a pathogen. The immune system is ordinarily brought back to a baseline response level after immune challenge through homeostatic processes in part regulated by the cytokines. Often, findings reported from various studies investigating the effects of stress on the cytokines are conflicting. This is due to some conditions has limited the accumulation of information on effects of cold stress on cytokine profile. The present study investigated the effects of multiple cytokines in response to a given stressor to understand the role of cytokines in the immunological responses to different duration of cold stress exposure in rats. Thus, researchers simulated animals' hypothermia life environment, using Luminex xMAP and evaluated the effect of cold stress for pro-inflammatory cytokines (IL-1 $\beta$, IL- 6 and TNF- $\alpha$ ), chemokine interferon- $\gamma$-inducible protein-10 (IP-10), Th1 (IL-2 and IFN- $\gamma$ ) and Th2 (IL-4 and IL-10) type of cytokine in serum of SPF Wistar rats. Irrespective of the duration, cold stress enhanced levels of proinflammatory cytokine $\mathbb{L}-6$. Acute cold stress up-regulated TNF- $\alpha$, IP-10, IL-2 and IL-4 levels. TNF- $\alpha$, IL-2 and IL-4 levels showed down-regulate trends compared with the acute cold stress treatment groups in the duration of chronic cold stress. Both IFN- $\gamma$ and IP-10 levels increased significantly in cold stress 9 and 12 days. These data demonstrate that cold stress enhances pro-inflammatory cytokines profile and alterations of Th1 and Th2 type cytokines secretion in serum of rats. With the extension of the cold stress time, the cellular adaptive immune response and to some extent, the nonspecific humoral immune response was also affected.
\end{abstract}

Key words: Cold stress, cytokines, Luminex xMAP, immune function, rat, China

\section{INTRODUCTION}

Animals will face various kinds of environmental stressors every day and cold stress as one of the stressors commonly exists in the cold region. Researchers have demonstrated that cold stress dramatically affected the health and welfare of animals in the cold region (Li et al., 2006; Wang et al., 2009; Yildirim and Yurekli, $2010 \mathrm{a}, \mathrm{b})$. Therefore, there is a need to understand how cold stressors affect health status of animals. Moreover, studies also proved that acute or chronic cold stress induces immunomodulatory effects in animal models as well as in human, mice and chicken (Banerjee et al., 1999; Brenner et al., 1999; Padgett and Glaser, 2003; Hangalapura et al., 2004).
Cold stress as a natural stressor may have its own unique pattern of neuroendocrine changes because of the accompanying body temperature variations that may influence immune functions (Shu et al., 1993). Cold stress affects various aspects of immune function depending on the nature and duration of the stress (Dhabhar, 2003). For example, stressors can directly affect the cells of the immune system and modulate the secretion of proinflammatory cytokines, Th1 or Th2 cytokines (Ruzek et al., 1997; O'Connor et al., 2003; Hangalapura et al., 2006). Cytokines play a key role in bidirectional communication between the neuro-endocrine and immune systems (Mahbub-E-Sobhani et al., 2008, 2011). It has been suggested that the interplay between

Corresponding Author: Huan Min Yang, College of Animal Science and Veterinary Medicine, 
hormones and cytokines during thermal stress may influence immune homeostasis in response to environmental challenges (Dugue, 2000; Russwurm et al., 2002). Most cytokines have pleiotropic, redundant and multifunctional functions and the level of one cytokine is tightly regulated by other cytokines (Adibhatla et al., 2008). Thus, some factors elevated by stress exposure could be responsible, in part for stress-induced suppression of other factors. It is important to examine multiple cytokines in response to a given stressor to understand the role of cytokines in the physiological response to cold stress exposure.

To date, there are a number of ways to administer cold stress and this may account for the different effects on the cytokines reported by various studies. However, there are some conditions had limited the accumulation of information on effects of cold stress on cytokine profile included the cold stimulus conditions, regulatory environment temperature, methods of cytokines detection and the choice of experimental animals in the studies. For example, some studies used cold water stress (Cheng et al., 1990; Shu et al., 1993; Dugue, 2000) which could not simulate animals' hypothermia life environment and the ambient temperature, humidity, carbon dioxide content could not be controlled precisely. Cold stress did not have a significant effect on IL-1 $\beta$ levels in man (Jansky et al., 1996) while in mice, cold exposure enhanced IL-1 cytokine levels (Cheng et al., 1990). Moreover, in order to correlate a specific physiological, pathology or disease process with changes environment temperature using traditional methodologies (real-time PCR, ELISPOT, ELISA and intracellular cytokine staining), limited sample sizes or budget restrictions can often prohibit this kind of testing (Hangalapura et al., 2006; Rhind et al., 2001).

In the present study, a climate room was described for precise regulation of temperature, humidity and carbon dioxide content. The precision of regulation for the temperature is $\pm 0.05^{\circ} \mathrm{C}$ and the relative humidity is $\pm 1 \%$. The homogeneous cold temperature could be obtained $\left( \pm 0.1^{\circ} \mathrm{C}\right)$ as cold stressor. To determine cytokine profiles in serum, one possible multiplex platform is the Luminex $\mathrm{xMAP}$, a bead array coupled with discrete fluorescent molecules to detect multiple soluble analytes (Wong et al., 2008). xMAP technology offers ideal speed and sensitivity for performing multiplexed cytokine measurements. Luminex's breakthrough approach offers comparable sensitivity to traditional ELISA-based systems (Elshal and McCoy, 2006) but with additional advantages including extended dynamic range and smaller sample size. With their small volume, low cost per test and multiplex capacity, Luminex-based cytokine assays have potential utility for present studies. The Specific Pathogen Free (SPF) rats were also a better choice in the studies which could reduce individual differences, to improve the accuracy of the experiment results.

The main aims of the present study was to determine the effect of different duration of cold stress (acute and chronic cold stress) on level of pro-inflammatory cytokines (IL-1 $\beta$, IL-6 and TNF- $\alpha$ ), chemokine interferon$\gamma$-inducible protein-10 (IP-10), Th1 (IL-2 and IFN- $\gamma$ ) and Th2 (IL-4 and IL-10) type of cytokines in serum of SPF Wistar rats.

\section{MATERIALS AND METHODS}

Treatment of animals: The study protocol was approved by the Animal Care Committee of Jilin University and was performed according to Chinese animal care guidelines. Rats were obtained from the Laboratory Animal Center, Academy of Military Medical Science. Ninety male SPF Wistar rats (12 weeks old, 190-240 g) were housed individually under diurnal lighting conditions at the climatic room (12-12 h light/dark) with free access to drinking water and a standard pellet diet. The environment in the climatic room was maintained at a temperature of $24 \pm 0.05^{\circ} \mathrm{C}$, relative humidity $45 \pm 0.1 \%$. The rats were divided into the following ten experimental groups: 1 group for the control group experiment, 4 groups for the acute cold stress experiment and 4 groups for the chronic cold stress experiment. The 8 stress treatment groups were maintained at $4 \pm 0.05^{\circ} \mathrm{C}(3,6,12$ and $24 \mathrm{~h}$ treatment groups for the acute cold stress and 3, 6, 9 and 12 days treatment groups for the chronic cold stress). After the treatments, rats are anesthetised by $75 \mathrm{mg} \mathrm{kg}^{-1}$ sodium pentobarbital. In order to determine cytokines levels in blood, $4 \mathrm{~mL}$ blood taken from the anesthesized rats by entering right ventricule from their hearts. The bloods were allowed to clot for at least $30 \mathrm{~min}$ before centrifugation for $10 \mathrm{~min}$ at $1000 \times \mathrm{g}$ and then remove serum and assay immediately or aliquot and store samples at $\leq-20^{\circ} \mathrm{C}$.

Cytokine measurements: Eight cytokines interleukin- $1 \beta$

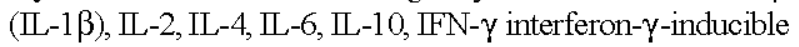
protein-10 (IP-10) and Tumor Necrosis Factor- $\alpha$ (TNF- $\alpha$ ) were measured using the MLLIPLEX MAP 8-plex Cytokine kit (Millipore, Billerica, MA) at the Institute of Pharmacology and Toxicology, Academy of Military Medical Science, Beijing, China. Median fluorescence intensity calculated from duplicates for each sample was collected using the Luminex-100 System Version 1.7 (Luminex). The StatLIA Software package (Ver. 3.2, Brendan Scientific Inc.) incorporating a weighted five-parameter logistic curve-fitting method was used to calculate sample cytokine concentrations. 
Statistical analysis: Statistical analysis was carried out using the SAS Version 9.1 statistical program. All data were expressed as arithmetic mean \pm standard deviation. One-way Analysis of Variance (ANOVA) tests were used to evaluate cytokine production across different cold stress time with overall significance for factors of cold stress time/concentration reported as such. Statistical significance was considered at the $5 \%$ level $(\mathrm{p}<0.05)$.

\section{RESULTS AND DISCUSSION}

Effect of cold stress on pro-inflammatory cytokines (IL-1 $\beta, \Pi L-6$ and TNF- $\alpha$ ) levels: The effects of cold stress on pro-inflammatory cytokine IL-1 $\beta$ levels were investigated in different cold stress time from $3 \mathrm{~h}$ to 12 days. As shown in Fig. 1, IL-1 $\beta$ levels had not significant difference among different duration of cold stress. As shown in Fig. 2, IL-6 levels increased significantly in cold stress 12 and $24 \mathrm{~h}, 3,6,9$ and 12 days treatment groups when compared to control group $(\mathrm{p}<0.05)$, $\mathbb{L}-6$ levels showed up-regulate volatility among different duration of cold stress. The concentration of TNF- $\alpha$ were upward trend in duration of acute cold stress. As shown in Fig. 3, acute cold stress significantly enhanced the concentration of TNF- $\alpha$ in stress 3, 6, 12 and $24 \mathrm{~h}$ treatment groups when compared to control group $(\mathrm{p}<0.05)$. The concentration of $\mathrm{TNF}-\alpha$ was significantly higher in acute cold stress $3,6,12$ and $24 \mathrm{~h}$ compared with chronic stress 6,9 and 12 days treatment groups $(\mathrm{p}<0.05)$.

Effect of cold stress on chemokine (IP-10) levels: The effects of cold stress on chemokine IP-10 levels were investigated in different cold stress time from $3 \mathrm{~h}$ to 12 days. As shown in Fig. 4, IP-10 levels increased significantly in $24 \mathrm{~h}$ cold stress group when compared to cold stress 3, 6, 12 and $24 \mathrm{~h}, 3$ and 6 days and control groups ( $p<0.05$ ). The levels of 9 and 12 days cold stress groups increased significantly compare with 3,6 and $12 \mathrm{~h}$, 3 and 6 days and control groups $(\mathrm{p}<0.05)$ but IP- 10 levels had not significant difference between 9 and 12 days cold stress groups.

Effect of cold stress on Th1 cytokines levels: In present study, we measure Thl cytokines (IL-2 and IFN- $\gamma$ ) levels in different duration of cold stress. The concentration of IL-2 was upward trend in duration of acute cold stress. Effects of acute and chronic cold stress on levels of the IL-2 are shown in Fig. 5. Compared with the corresponding control group, acute cold stress in $3,6,12$ and $24 \mathrm{~h}$ treatment groups significantly increased

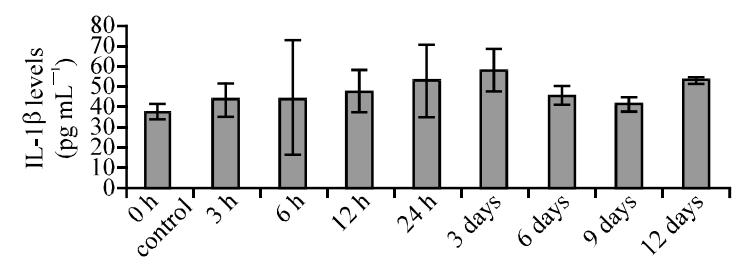

Fig. 1: The effect of different duration of cold stress treatment on interleukin-1 $\beta$ levels in rat serum. Analysis of variance followed by one-way Analysis of Variance tests

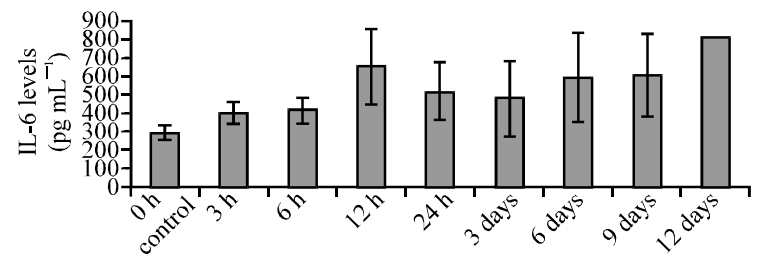

Fig. 2: The effect of different duration of cold stress treatment on interleukin- 6 levels in rat serum. Analysis of variance followed by one-way analysis of variance tests

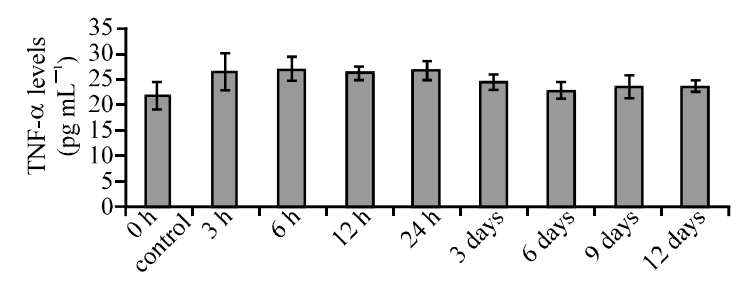

Fig. 3: The effect of different duration of cold stress treatment on TNF- $\alpha$ levels in rat serum. Analysis of variance followed by one-way analysis of variance tests

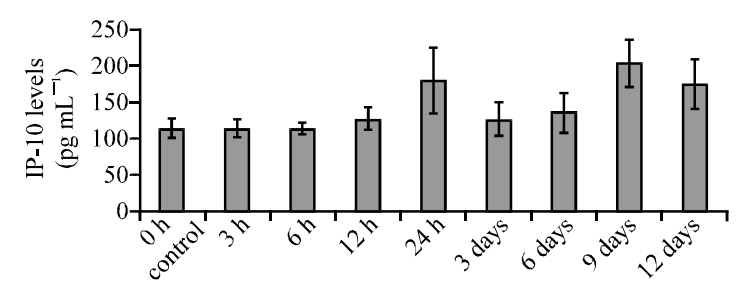

Fig. 4: The effect of different duration of cold stress treatment on Interferon- $\gamma$-inducible protein-10 levels in rat serum. Analysis of variance followed by one-way Analysis of Variance tests

$(\mathrm{p}<0.05)$. IL-2 level of 3 days chronic cold stress group was higher significantly than control group $(\mathrm{p}<0.05)$ but there were not difference significantly in the other chronic stress groups. IL-2 levels returned to normal 
concentration in chronic stress groups from 6-12 days compared with control group. As shown in Fig. 6 , IFN- $\gamma$ level increased significantly in chronic cold stress 9 and 12 days treatment groups when compared to any other acute or chronic cold stress treatment groups $(\mathrm{p}<0.05)$. IFN- $\gamma$ levels were higher significantly in the 9 days cold stress compared with 12 days cold stress treatment group $(\mathrm{p}<0.05)$. Compared with the corresponding control group, acute cold stress in 12 and $24 \mathrm{~h}$ treatment groups significantly increased $(\mathrm{p}<0.05)$.

Effect of cold stress on Th2 cytokines levels: Th2 cytokines (IL-4 and IL-10) levels were investigated in different cold stress time from $3 \mathrm{~h}$ to 12 days. As shown in Fig. 7, cold stress significantly enhanced the concentration of IL-4 in stress 6 and $24 \mathrm{~h}, 3$ and 6 days treatment groups when compared to control group $(p<0.05)$. As shown in Fig. 8, IL-10 levels have not significant difference among different duration of cold stress.

The reactive mechanism of cold stress is very complicated and the results from different studies are not consistent. These may be attributed to the duration of cold exposure, the temperature of cold exposure, the genetic background of experimental animal and so on. But lots of studies have proved that cold stress could influence the energy metabolism and immune responses (Rybakina et al., 1997; Cichon et al., 2002; Haman et al.,

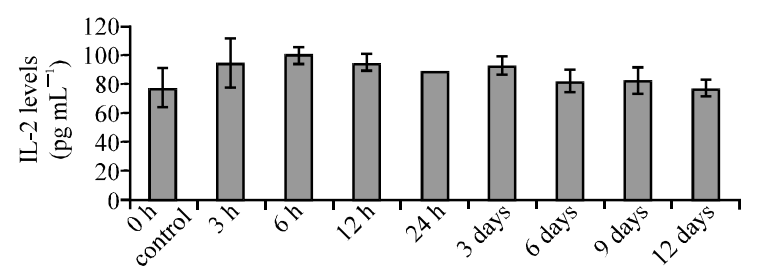

Fig. 5: The effect of different duration of cold stress treatment on interleukin-2 levels in rat serum. Analysis of variance followed by one-way Analysis of Variance tests

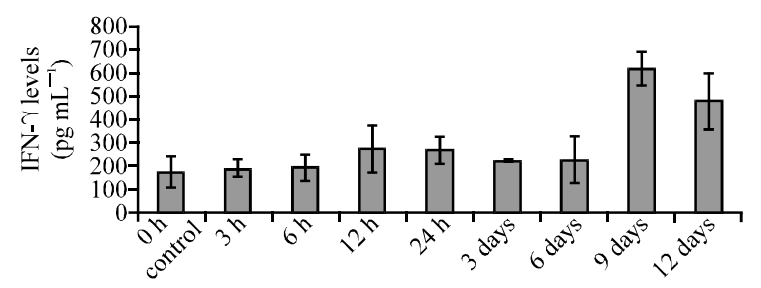

Fig. 6: The effect of different duration of cold stress treatment on IFN- $\gamma$ levels in rat serum. Analysis of variance followed by one-way Analysis of Variance tests
2002). Pro-inflammatory cytokines are important in the recruitment of immune cells to the site of infection. Production pro-inflammatory cytokines can be stimulated directly by depression and other negative emotions and stressful experiences. Indeed, both physical and psychological stressors can provoke transient increases in pro-inflammatory cytokines (Glaser and Kiecolt-Glaser, 2005). In the present study, researchers examined proinflammatory cytokines $\amalg-1 \beta, \mathrm{IL}-6$ and TNF- $\alpha$ which were the principle cytokines that mediate acute inflammation. Irrespective of the duration, cold stress did not induct the change significantly of IL-1 $\beta$ levels, IL-1 $\beta$ levels showed slightly up-regulate trend in acute cold stress from 3-24 h and still showed increase to chronic cold stress 3 days. The results of IL-1 $\beta$ were consistent with man underwent cold and wet exposures (Jansky et al., 1996). While in mice and chicken, cold exposure enhanced significantly IL-1 $\beta$ levels (Cheng et al., 1990; Hangalapura et al., 2006).

Cold stress up-regulated expression of both IL-6 and TNF- $\alpha$ and this effect was determined by the duration of cold stress. With the strengthening of the cold stress (from acute cold stress to chronic cold stress), IL-6 levels still showed increase trend. Many studies have also demonstrated that restraint stress could induce elevation of the plasma IL-6level. Yildirim and Yurekli (2010a) found that cold stress induced the increase of IL- 6 levels in rat liver, lung and brain and heart tissues. Takaki et al. (1994) found that immobilization stress may increase plasma IL-6 via central and peripheral catecholamines (Nukina et al.,

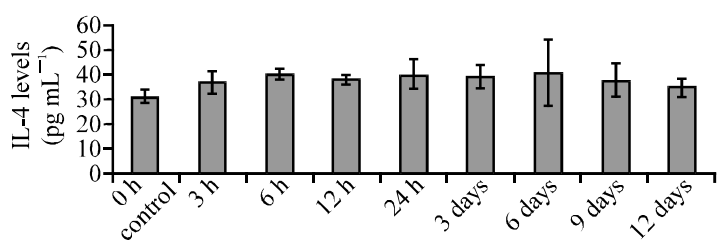

Fig. 7: The effect of different duration of cold stress treatment on interleukin-4 levels in rat serum. Analysis of variance followed by one-way Analysis of Variance tests

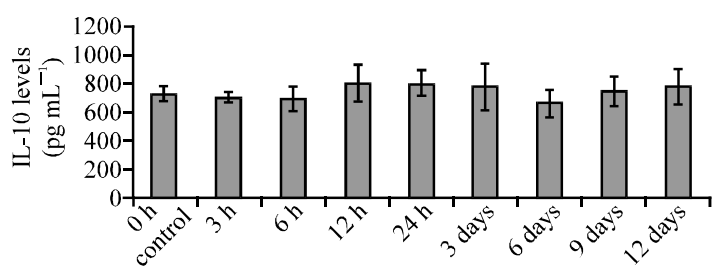

Fig. 8: The effect of different duration of cold stress treatment on interleukin-10 levels in rat serum. Analysis of variance followed by one-way Analysis of Variance tests 
2001). Brenner examined the human immunological responses to cold exposure and they found a rise in circulating levels of IL-6 (Brenner et al., 1999).

Acute cold stress induced the increase significantly of TNF- $\alpha$ but TNF- $\beta$ showed down-regulate trend in chronic cold stress from 3-12 days until the concentration of TNF- $\alpha$ down to normal range compared with control group. So, researchers predicted that TNF- $\alpha$ could do a stress maker in the duration of acute cold stress. To date, IL-1, IL- 6 and TNF- $\alpha$ cytokines are the major proinflammatory cytokines that are responsible for early response of inflammation and either act as endogenous pyrogens (Netea et al., 2000). They help protect against infection and prepare injured tissue for repair by enhancing phagocytic cell recruitment and activation. Furthermore, pro-inflammatory cytokines released by recruited cells regulate the ability of fibroblasts and epithelial cells to remodel the damaged tissue (Kiecolt-Glaser et al., 1995). In the present study, the oversecretion of pro-inflammatory cytokines might to reduce the damage caused by cold stress.

To date, the patients with primary Sjogren's syndrome showed increased IL-6 levels of serum were correlated with poor quality of life in these individuals and the study suggested that the patients with concentrations of IL-6 higher than those of the healthy controls showed a significantly lower score in the dimensions of bodily pain and physical functioning (Baturone et al., 2009). So, researchers predicted that IL-6 levels increased significantly from acute to chronic cold stress compared with control group which may be related to against the pain and damage caused by cold stress. Furthermore, Stevenson suggested that IL-6 was an important early indicator in evaluating piglet health status (Stevenson et al., 2006). Andrew analyzed the levels of cytokines after psychological stress and they found that IL-6 was the most representative indicator (Steptoe et al., 2007). Thus, we suggested that IL-6 could do as a hallmark cytokine when rats were in the environmental challenges.

Cytokines produced by the innate immune system lead to differentiation of the T-helper 1 (Th1) and 2 (Th2) immune pathways. Activation of Thl often involves stimulation of cellular immunity while Th2 is associated with humoral immunity. These results of present study, together with changes in IL- 6 and TNF- $\alpha$ level, imply that cold stress may result in dysregulation of the Th1/Th2 cytokine profiles break the Th1/Th2 balances and then affect immune response.

The Thl response is associated with the release of the cytokines IFN- $\gamma$ and $\mathbb{L}-2$. IFN- $\gamma$ is a key cytokine which is central to stimulatory and inhibitory roles of a Th subset. IFN- $\gamma$ does not directly inhibit differentiated Th2 cells. Instead, they were inhibited by blocking the differentiation of these subsets from naive precursors. Essentially, IFN- $\gamma$ has been shown to inhibit Th2 response (Coffman, 2006). In this present study, we also found that IFN- $\gamma$ and IP-10 had similar trends in duration of cold stress. Yue et al. (2011) found that elevated IFN- $\gamma$ concentration could induce macrophages to secrete the IP-10. IP-10 is the production of the Th1-attracting chemokines which preferentially promotes Thl immune responses (Yue et al., 2011). The results suggest that IFN- $\gamma$ and IP-10 also had synergic action to promote cellmediated immunity in the duration of cold stress.

IL-2 level is an important indicator of cellular immunity (Chen et al., 2011). In the present study, IL-2 levels increased significantly in acute cold stress treatment groups compared with control group. The result would increase an evidence indicates that acute cold stress could have a stimulating effect on cell-mediated immunity which is consistent with study results of Dhabhar and McEwen (1996) (Dhabhar et al., 1996; Jiang et al., 2007). To date, mild stress or hormone related with stress treatment to animals, the immune level of animals would be enhanced. Numerous studies showed that small amounts of harmful or cold stressful agents could be beneficial for the health of laboratory animals and improve immunity (Dhabhar et al., 1996; Dhabhar and McEwen, 1999). In this present study, IL-2 levels returned to normal concentration compared with control group in the duration of chronic cold stress which indicated that the body has adapted gradually to the cold stress. But with the strengthening of the cold stress and immunosuppression would be induced. Rhind found that cold stress enhanced concentration of IL- 6 , IL-2 and TNF- $\alpha$ in human serum (Rhind et al., 2001). Liu Ya-Li suggested that oral (gavage) administration of compound nutrients was found to attenuate the acute and chronic immobilization and cold water immersion stress-induced increase in serum IL-6 levels and decrease in IL-2 levels (Liu et al., 2007). The result of IL-2 was converse to the present study. It was showed that cold stressors can directly affect the secretion of cytokines, under different stress conditions, cytokines showed different trends.

The Th2 response is associated with the release of IL-4 and IL-10. These cytokines tend to enhance the production of antibodies. To the data showed that cold stress up-regulated the IL-4 levels from duration of cold stress $24 \mathrm{~h}$ to 6 days and did not affect IL-10 levels. IL-4 is a key regulator in humoral and adaptive immunity and decreases the production of Th1 cells and macrophages. IL-4 antagoizes the effects of IFN- $\gamma$ and thus inhibits cell-mediated immunity (Coccia et al., 2000). IL-4 as the 
hallmark cytokine of Th2 immunity if it is overexpressed, it negatively interferes with the immune defense mechanisms thus decreasing the recruitment, expansion or activity of major effector cells such as the Th1 cells (Salak-Johnson and McGlone, 2007). In the duration of chronic cold stress from 3-6 days, IL-4 and IL-6 (Th2 related) levels sharply higher compared with the other treatment groups, IL-2 and IFN- $\gamma$ (Th1 related) levels returned to normal range which indicated that humoral immunity was becoming dominant.

Acute and chronic stressors tend to affect the immune responses differently whereby chronic stress most often leads to suppression of the immune system. Often, acute stress has limited suppressive effects on immune function (Salak-Johnson and McGlone, 2007). To the knowledge, the data provided herein are the first to examine whether different duration of cold stress affects the role of multiple cytokines in the response (s) to cold stressor exposure in rats.

\section{CONCLUSION}

In this study, acute cold stress enhanced the immune level of rats. When cold stress was prolonged, the cellular adaptive immune response and to some extent, the nonspecific humoral immune response was also affected. These results suggest that eight cytokines reflects archetypal Th1- and Th2-type responses and provide insight regarding the polarization of cytokine activity at different cold stress levels. Moreover, the underlying factors seem to be estimable and can be used to represent divergent profiles in cold stress analyses. However, it should be emphasized that none of the cytokines specific to one particular subset are exclusive products of Th cells because other leukocytes can contribute to Th1 or Th2type responses. It is important to point out that like many other researchers, we studied only one specific part of the immune function. We lack information on whether the function of the whole immune system was affected by cold stress. Clearly, the pattern of multiple cytokines induction and the role of multiple cytokines in the response (s) to cold stressor exposure require further attention.

\section{ACKNOWLEDGEMENTS}

This research is supported by the National Natural Science Foundation of China (Grant No.: 30972159), Special Fund for Agro-Scientific Research in the Public interest, China (Grant No.: 2010030360-03-3) and the National 948 Project of China (Grant No. 2011-G35).

\section{REFERENCES}

Adibhatla, R.M., R. Dempsey and J.F. Hatcher, 2008. Integration of Cytokine Biology and lipid metabolism in stroke. Front Biosci., 13: 1250-1270.

Banerjee, S.K., H. Aviles, M.T. Fox and F.P. Monroy, 1999. Cold stress-induced modulation of cell immunity during acute Toxoplasma gondiiinfection in mice. J. Parasitol., 85: 442-447.

Baturone R., M.J. Soto, M. Marquez, 2009. Health-relaed quality of life in patients with primary Sjogren's syndrome: Relationship with serum levels of proinflammaory cytokines. Scand J. Rheumatol., 38: 386-389.

Brenner, I.K., J.W. Castellani, C. Gabaree, A.J. Young, J. Zamecnik, R.J. Shephard and P.N. Shek, 1999. Immune changes in humans during cold exposure: Effects of prior heating and exercise. J. Applied Physiol., 87: 699-710.

Chen, J., Y.X. Zhou, X.D. Jin and S.W. Chen, 2011. Clinical experience: Expression of interleukin-2 in candidal balanoposthitis and its clinical significance. Chin. Med. J., 124: 2776-2778.

Cheng, G.J., J.L. Morrow-Tesch, D.I. Beller, E.M. Levy and P.H. Black, 1990. Immunosuppression in mice induced by cold water stress. Brain Behav. Immun., 4: 278-291.

Cichon, M., M. Chadzinska, A. Ksiazek and M. Konarzewski, 2002. Delayed effects of cold stress on immune response in laboratory mice. Proc. Biol. Sci., 269: 1493-1497.

Coccia, E.M., E. Stellacci and G. Marziali, 2000. IFN- $\gamma$ and I-4 differently regulate inducible NO synthase gene expression through IRF-1 modulation. Int. Immunol., 12: $977-985$.

Coffman, R.L., 2006. Origins of the Th1-Th2 model: A person perspective. Nat. Immunol., 7: 539-541.

Dhabhar, F.S. and B.S. McEwen, 1996. Stress-induced enhancement of antigen-specific cell-mediated immunity. J. Immunol., 156: 2608-2615.

Dhabhar, F.S. and B.S. Mcewen, 1999. Enhancing versus suppressive effects of stress hormones on skin immune function. Proc. Natl. Acad. Sci., 96: 1059-1064.

Dhabhar, F.S., 2003. Stress, leukocyte trafficking, and the augmentation of skin immune function. Ann. N.Y. Acad. Sci., 992: 205-217.

Dhabhar, F.S., A.H. Miller and B.S. McEwen, 1996. Stress-induced changes in blood leukocyte distribution. Role of adrenal steroid hormones. J. Immunol., 157: 1638-1644. 
Dugue, L., 2000. Adaptation related to cytokines in man: Effects of regular swimming in ice-cold water. Clin. Physiol., 20: 114-121.

Elshal, M.F., J.P. McCoy, 2006. Multiplex bead array assays: Performance evaluation and comparison of sensitivity to ELISA. Methods, 38: 317-323.

Glaser, R., J.K. Kiecolt-Glaser, 2005. Stress-induced immune dysfunction: Implications for health. Nat. Rev. Immunol., 5: 243-251.

Haman, F., F. Peronnet, G.P. Kenny, D. Massicotte, C. Lavoie, C. Scott and J.M. Weber, 2002. Effect of cold exposure on fuel utilization in humans: Plasma glucose, muscle glycogen and lipids. J. Applied Physiol., 93: 77-84.

Hangalapura, B.N., M.G. Kaiser, J.J. van der Poel, H.K. Parmentier and S.J. Lamont, 2006. Cold stress equally enhances in vivo pro-inflammatory cytokine gene expression in chicken lines divergently selected for antibody responses. Dev. Comp. Immunol., 30: $503-511$.

Hangalapura, B.N., M.G. Nieuwland, G. de Vries Reilingh, H. van den Brand, B. Kemp and H.K. Parmentier, 2004. Durations of cold stress modulates overall immunity of chicken lines divergently selected for antibody responses. Poult. Sci., 83: 765-775.

Jansky, L., D. Pospisilova, S. Honzova, B. Ulicny, P. Sramek, V. Zeman and J. Kaminkova, 1996. Immune system of cold-exposed and cold adapted humans. Eur. J. Appl. Physiol. Occup. Physiol., 72: 445-450.

Jiang, D.M., S.Z. Li and H.M. Yang, 2007. Effect of cold stress on artery pressure and serum cytokines of rabbits. J. Environ. Health, 24: 577-579.

Kiecolt-Glaser, J.K., P.T. Marucha, W.B. Malarkey, A.M. Mercado and R. Glaser, 1995. Slowing of wound healing by psychological stress. Lancet, 346: 1194-1196.

Li, S.Z., B.B Ren., H.M. Yang, Y.Y. Yang, H. Ji and J. Ni, 2006. Effect of cold stress with different intensities on HSP70 expression in Wistar rat muscle, spleen and liver. Chin. J. Applied Environ. Biol., 12: 235-238.

Liu Y.L., B. Hui, S.M. Chi, R.Fan and Y.M. Wang et al., 2007. The effect of compound nutrients on stressinduced changes in serum IL-2, IL- 6 and TNF- $\alpha$ levels in rats. Cytokine, 37: 14-21.

Mahbub-E-Sobhani, N. Haque, A.T.M.K. Islam, U. Salma, A. Ahmed, I.J. Mukti and A.K.M.F. Haque, 2008. Brief naturalistic stressors cause shift of $\mathrm{TH} 1$ to $\mathrm{TH} 2$ cytokine response and increase disease susceptibility. Biotechnology, 7: 623-629.

Mahbub-E-Sobhani, N. Haque, U. Salma and A. Ahmed, 2011. Immune modulation in response to stress and relaxation. Pak. J. Biol. Sci., 14: 363-374.
Netea, M.G., B.J.Kullberg and J.W. Van der Meer, 2000. Circulating cytokines as mediators of fever. Clin. Infect. Dis., 31: 178-184.

Nukina, H., N. Sudo, Y. Aiba, N. Oyama, Y. Koga and C. Kubo, 2001. Restraint stress elevates the plasma interleukin-6 levels in germfree mice. J. Neuroimmunol., 115: 46-52.

O'Connor, K.A., J.D. Johnson, M.K. Hansen, J.L. Wieseler Frank, E. Maksimova , L.R. Watkins and S.F. Maier, 2003. Peripheral and central proinflammatory cytokine response to a severe acute stressor. Brain Res., 991: 123-132.

Padgett, D.A. and R. Glaser, 2003. How stress influences the immune response. Trends Immunol., 24: 444-448.

Rhind, S.G., J.W. Castellani, I.K. Brenner, R.J. Shephard and J. Zamecnik et al., 2001. Intracellular monocyte and serum cytokine expression is modulated by exhausting exercise and cold exposure. Am. J. Physiol. Regul. Integr. Comp. Physiol., 281: 66-75.

Russwurm, S., I. Stonans, K. Schwerter, E. Stonane, W. Meissner and K. Reinhart, 2002. Direct influence of mild hypothermia on cytokine expression and release in cultures of human peripheral blood mononuclear cells. J. Interferon Cytokine Res., 22: 215-221.

Ruzek, M.C., A.H Miller, S.M. Opal, B.D. Pearce and C.A. Biron, 1997. Characterization of early cytokine responses and an interleukin (IL)-6-dependent pathway of endogenous glucocorticoid induction during murine cytomegalovirus infection. J. Exp. Med., 185: 1185-1192.

Rybakina, E.G., S.N. Shanin, I.A. Kozinets, E.E. Fomicheva and E.A. Korneva, 1997. Cellular mechanisms of cold stress related immunosuppression and the action of interleukin 1. Int. J. Tissue React., 19: 135-140.

Salak-Johnson, J.L. and J.J. McGlone, 2007. Making sense of apparently conflicting data stress and immunity in swine and cattle. J. Anim. Sci., 85: 81-88.

Shu, J., J.R. Stevenson and X. Zhou, 1993. Modulation of cellular immune responses by cold water swimm stress in the rat. Dev. Comp. Immunol., 17: 357-371.

Steptoe, A., M. Hamer and Y. Chida, 2007. The effects of acute psychological stress on circulating inflammatory factors in human: A review and metaanalysis. Brain Behav. Immun., 21: 901-912.

Stevenson, L.S., K. McCullough, I. Vincent, D.F. Gilpin and A. Summerfield, 2006. Cytokine and C-reactive protein profiles induced by porcine circovirus type 2 experimental infection in 3-week-old piglets. Viral Immunol., 19: 189-195. 
Takaki, A., Q.H. Huang, A. Somogyvari-Vigh and A. Arimura, 1994. Immobilization stress may increase plasma interleukin-6 via central and peripheral catecholamines. Neuroimmunomodulation, 1:335-342.

Wang, J.T., S. Li, J.L. Li, J.W. Zhang and S.W. Xu, 2009. Effects of cold stress on the messenger ribonucleic acid levels of peroxisome proliferator-activated receptor-in spleen, thymus and bursa of Fabricius of chickens. Poult. Sci., 88: 2549-2554.

Wong, H.L., R.M. Pfeiffer, T.R. Fears, R. Vermeulen, S. Ji and C.S. Rabkin, 2008. Reproducibility and correlations of multiplex cytokine levels in asymptomatic persons. Cancer Epidemiol. Biomarkers Prev., 17: 3450-3456.
Yildirim, N.C. and M. Yurekli, 2010b. Stress-induced changes in tyrosine hyroxylase enzyme activity and adrenomedullin levels in rat hypothalamus, adrenal medulla and heart tissues. Asian J. Anim. Vet. Adv., 5: 339-345.

Yildirim, N.C. and M. Yurekli, 2010a. The effect of adrenomedullin and cold stress on interleukin-6 levels in some rat tissues. Clin. Exp. Immunol., 161: 171-175.

Yue, Y., J. Gui, W. Ai, W. Xu and S. Xiong, 2011. Direct gene transfer with IP-10 mutant ameliorates mouse CVB3-induced myocarditis by bluting Th1 immune responses. PLoS One, 6: 181-186. 